\title{
Toward Detection of Electron-Hole Pair Excitation in H-atom Collisions with Au(111): Adiabatic Molecular Dynamics with a Semi-Empirical Full-Dimensional Potential Energy Surface
}

\author{
By Svenja M. Janke ${ }^{*}$, Michele Pavanello ${ }^{2}$, Geert-Jan Kroes ${ }^{3}$, Daniel Auerbach ${ }^{4}$, \\ Alec M. Wodtke ${ }^{1}$, and Alexander Kandratsenka ${ }^{1, *}$ \\ ${ }^{1}$ Institute for Physical Chemistry, Georg-August University of Göttingen, Tammannstr. 6, 37077, \\ Germany \\ 2 Rutgers University, Warren St. 73, Newark, NJ, 07102, USA \\ ${ }^{3}$ Leiden Institute of Chemistry, Gorlaeus Laboratories, Leiden University, 2300, The Netherlands \\ ${ }^{4}$ Max-Planck-Institute for Biophysical Chemistry, Faßberg 11, 37077, Germany
}

(Received March 9, 2013; accepted in revised form June 2, 2013)

(Published online August 5, 2013)

\section{H-atom Scattering / Effective Medium Theory / Potential Energy Surface / MD Simulations}

We report an analytic potential energy surface (PES) based on several hundred DFT energies for $\mathrm{H}$ interacting with a $\mathrm{Au}(111)$ surface. Effective medium theory is used to fit the DFT data, which were obtained for the Au atoms held at their equilibrium positions. This procedure also provides an adequate treatment of the PES for displacements of Au atoms that occur during scattering of $\mathrm{H}$ atoms. The fitted PES is compared to DFT energies obtained from ab initio molecular dynamics trajectories. We present molecular dynamics simulations of energy and angle resolved scattering probabilities at five incidence angles at an incidence energy, $E_{\mathrm{i}}=5 \mathrm{eV}$, and at a surface temperature, $T_{\mathrm{S}}=10 \mathrm{~K}$. Simple single bounce trajectories are important at all incidence conditions explored here. Double bounce events also make up a significant fraction of the scattering. A qualitative analysis of the double-bounce events reveals that most occur as collisions of an H-atom with two neighboring surface gold atoms. The energy losses observed are consistent with a simple binary collision model, transferring typically less than $150 \mathrm{meV}$ to the solid per bounce.

\section{Introduction}

In this paper, we initiate an effort to find an atomic-scale, quantitative description of the forces defining $\mathrm{H}$ atom $(\mathrm{H})$ interactions with a metal $(\mathrm{M})$ at its surface, within the subsurface region and within the bulk. $\mathrm{H} / \mathrm{M}$ interactions might appear to be a simple (even trivial) problem in chemical physics. But appearances can deceive. Indeed, this class

* Corresponding authors. E-mails: sjanke@gwdg.de; akandra@gwdg.de 
of problems exhibits a dynamical richness, which makes it a particularly interesting as well as challenging target for theoretical simulations, not to mention experiment. Due to the highly directional chemical interactions between $\mathrm{H}$ and $\mathrm{M}$, the potential energy surface (PES) can be highly corrugated [1]. Strong chemical interactions of H-atoms with most metals (characterized by surface binding energies in the range $-2 \mathrm{eV}$ to $-4 \mathrm{eV}$ ) [2] also mean that in $\mathrm{H}$-atom collisions at surfaces, the $\mathrm{H}$-atom is accelerated with at least $1-2 \mathrm{eV}$ translational energy prior to impact. Due to their small mass, H-atoms dissipate this energy inefficiently to phonons. These factors suggest the existence of (as yet unobserved) hot-atom intermediates, which have been invoked to explain several peculiar observations in surface chemistry $[3,4]$. Another interesting characteristic of the $\mathrm{H}$-atom is its size; the $\mathrm{H}$-atom is small. Hence, energetic barriers between surface and sub-surface $\mathrm{H}$-atom binding sites can be low [2,5-7]. The energetics of H-atom bonding in metals is also strongly influenced by lattice relaxation, especially for sub-surface binding sites $[1,8]$. Hence, an accurate treatment of the dynamics requires a high dimensional PES, capable of describing metal atom (lattice) motion. Finally, there is evidence that H-atom collisions at metals can lead to electron-hole pair (EHP) excitation [914]. Thus, an accurate description of electronically non-adiabatic effects may also be an important aspect of this problem $[15,16]$.

An analytical full-dimensional PES would be useful for molecular dynamics studies, since simulations could be performed with even millions of trajectories. For example, one could make specific predictions within (and even beyond) the electronically adiabatic approximation concerning angle and energy resolved $\mathrm{H}$-atom scattering experiments, which could be carried out in the future. Furthermore, with this approach the description of phonon excitation in the $\mathrm{H}$-atom collision is almost unrestricted with respect to unit-cell size or simulation time. Hence, an analytical full dimensional PES will also be suitable for simulations of $\mathrm{H}$-atom trapping, which may require simulation times of up to several ps. One may even imagine that such a full dimensional PES could be used to describe bulk diffusion [15-18].

In this paper, we report a new PES for H-atoms interacting with an $f c c$ gold crystal at its (111) surface and present preliminary results of classical MD calculations. Density functional theory (DFT) with a generalized gradient approximation (DFT-GGA) employing a mixed functional, i.e., the functional that is the specific reaction parameter (SRP) density functional for $\mathrm{H}_{2}+\mathrm{Cu}(111)$ [19,20], is used to calculate energies on the PES for a large number of chosen positions of the H-atom within, on the surface and outside of the frozen $f c c$ Au-lattice. These data are then fit to an analytic function derived from effective medium theory (EMT) [21-23]. The EMT expression for energy describes by definition the $\mathrm{Au} \mathrm{Au}$ interatomic forces in a realistic albeit semiempirical way, even when the Au atoms are not in their equilibrium positions. Hence, this approach leads to a full dimensional PES capable of describing energy transfer from $\mathrm{H}$-atom translation to Au-atom motion. The protocol presented here has been previously employed to investigate $H$ on Copper [1] and $H$ on Palladium [15,16]. Furthermore, to ensure the validity of our EMT-based PES, we compare how well our PES reproduces energy values along ab initio molecular dynamics (AIMD) trajectories.

We follow an approach to the $\mathrm{H} / \mathrm{M}$ interaction that is fully within the spirit of Eyring and Polanyis seminal paper [24] highlighted in this issue. Since PESs were first introduced by Eyring and Polanyi to study gas phase reactions, they have proven them- 
selves also useful to analyze the interaction of molecules with metal surfaces, a seminal early example being the PES cut describing the interaction of $\mathrm{H}_{2}$ with a copper surface presented by Lennard-Jones in his 1932 paper on adsorption and diffusion on solid surfaces [25]. PESs have also been tremendously useful for studying reactions of molecules on metal surfaces [19,26-37]. Many experimental trends can be understood, and in some cases quantitative agreement with experiment may be obtained, even if electron-hole pair excitation or energy transfer to surface phonons is neglected in these studies. This is not a trivial finding. For instance, experiments have shown that scattering of atoms and molecules from metal surfaces may lead to considerable electron-hole (e-h) pair excitation for systems with deep atomic or molecular chemisorption wells [14], and that scattering of highly vibrationally excited NO from low work function metal surfaces may lead to vibrationally promoted electron emission [38].

Here, we describe the development of a preliminary high-dimensional potential energy surface, based on a mixture of first principles and semi-empirical ideas that is capable of realistically describing the high energy scattering of an H-atom in the presence of a moving crystalline Au-metal lattice. We demonstrate classical molecular dynamics (MD) results that characterize scattering from the surface. With this approach hundreds of thousands of classical trajectories can be calculated easily and we may simulate energy and angle resolved scattering within the electronically adiabatic approximation with high statistical accuracy.

Specifically, we use this new PES to carry out MD simulations for collisions of $\mathrm{H}$-atoms with $\mathrm{Au}(111)$ within the electronically adiabatic approximation at five incidence conditions with $E_{\mathrm{i}}=5 \mathrm{eV}$ and $T_{\mathrm{S}}=10 \mathrm{~K}$. Simple, single bounce trajectories can be identified and are an important contribution to the overall scattering at all incidence conditions. They exhibit an energy loss that can be understood from the attractive Baule limit [39]. Back scattered single bounce trajectories travelling nearly parallel to the surface transfer less than $2 \%$ of the $\mathrm{H}$-atom incidence energy to Au atom motion. Double (and higher) bounce collisions are important at all incidence conditions examined in his work. The energy loss due to double bounce trajectories is consistent with a value twice the attractive Baule limit. The majority of double bounce trajectories are H-atom collisions with two neighboring surface gold atoms; however, a remarkably large number of double bounce trajectories $(25 \%)$ are long range in nature, where the second collision is farther away than the next nearest neighbor. The remainder of the paper is organized as follows. In Sect. 2 we describe the methods used in this work, which include sections on our implementation of DFT using the VASP package, our fitting method employing EMT and a description of our molecular dynamics approach, including ab initio molecular dynamics. In Sect. 3 we present results. We describe the new multi-dimensional PES, we evaluate its accuracy and we present results of MD calculations. Section 4 gives a brief discussion with conclusions.

\section{Methods}

\subsection{Electronic structure calculations}

To construct a potential energy surface, we have first calculated 560 points with density function theory (DFT) [40,41], making use of the ab initio simulation package 
VASP [42-45]. In this environment, we used ultra-soft pseudo potentials and the mixed GGA functional that was implemented into the VASP code to get an optimal description of the $\mathrm{H}_{2}+\mathrm{Cu}(111)$ system. The functional used is a mixture of the PBE [46] (coefficient 0.52) and RPBE (coefficient 0.48) functional, and may be viewed as an adaptation of the SRP density functional first designed [19] for the description of the reactive scattering of $\mathrm{H}_{2}$ from $\mathrm{Cu}(111)$ for use with VASP (for details, see Ref. [20]). From now on, we refer to this functional as the R48PBE functional, to express that it is a weighted average of the RPBE and PBE functionals, with a weight of 0.48 for the RPBE functional. Because this functional yields a chemically accurate description of the $\mathrm{H}_{2}+\mathrm{Cu}(111)$ system $[19,20]$ we also expect it to be accurate for $\mathrm{H}+\mathrm{Cu}(111)$ and, by extension, for the interaction of $\mathrm{H}$ with the (111) surface of the noble metal considered here $(\mathrm{Au})$. This is also in line with previous calculations on $\mathrm{H}$-atoms interacting with small Au-clusters, which used both $a b$ initio and DFT methods [47]. These calculations showed that the PW91 functional [48], which yields energies quite similar to PBE results [42-45], yields $\mathrm{H}$-Au cluster interaction energies that already compare reasonably well with $\operatorname{CCSD}(\mathrm{T})$ results, while the results should be improved by mixing in the RPBE functional [47], as done here.

For the calculations presented here, we used a $8 \times 8 \times 1 k$-point mesh and a cut-off energy of $300 \mathrm{eV}$ for the plane wave expansion. We constructed our theoretical Au lattice by standard methods. Briefly, we first carry out DFT calculations with 3D periodic boundary conditions to simulate bulk $f c c$ gold. This gave a relaxed lattice constant of $4.201 \AA$, which may be compared to the experimental value of $4.08 \AA$. Subsequently we constructed a four layer slab in a $2 \times 2$ cell. We fixed the $\mathrm{Au}-\mathrm{Au}$ distances within each layer (to be $2.971 \AA=4.201 \AA / \sqrt{2}$ ) and let the interlayer distance relax, holding the distance between the two lowest layers fixed at $(2.425 \AA=4.201 \AA / \sqrt{3})$. This resulted in interlayer relaxation distances of $\approx 0.02 \AA$. We used this structure in a $3 \times 3$ 4-layer slab to ensure that there is no interaction between the hydrogen atoms in neighboring super cells. We perform DFT calculations for 560 different positions of the $\mathrm{H}$ atom. Figure 1 shows the 10 symmetry sites normal to which we calculated DFT energies at 560 values of $z$ (the Cartesian coordinate normal to the surface). Note that we placed a $13 \AA$ thick vacuum layer between the slabs defining the periodic boundary conditions along the $z$-axis. Figure 2 shows all of these 560 DFT energies as black filled circles.

\subsection{Fitting the energy points: effective medium theory}

The fitting according to the Effective Medium Theory (EMT) was performed following the approach of Nørskov et al. [23] and Strömquist et al. [1] as it is implemented in the Atomic Simulation Environment (ASE) by CAMd from the Technical University of Denmark [49]. The idea behind the EMT is that one first calculates the energy of a reference system, the effective medium. One then models the difference between the reference system and the actual system of interest. In this work, we choose a perfect $\mathrm{Au}$ crystal as the reference system. The interaction potential developed to describe a binary metal compound with a hydrogen atom and a gold lattice considered as an adsorbate 
a)
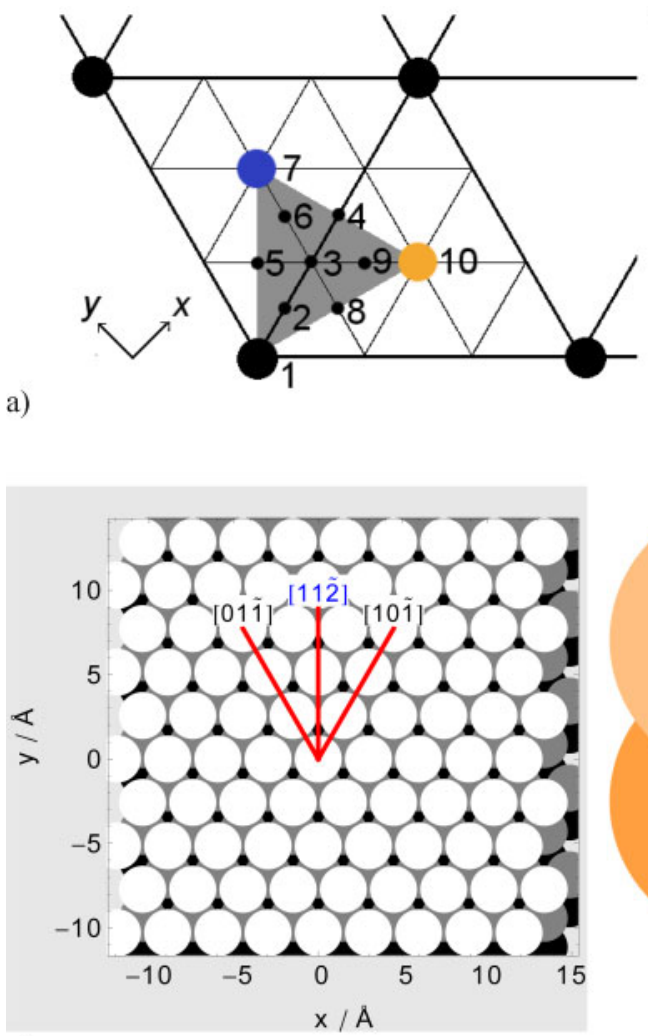

c)

Fig. 1. Geometric Definition of the Potential Energy Surface. DFT calculations of the energy of an H-atom in the presence of an $f c c$ gold lattice were carried out along lines normal to the $\mathrm{Au}(111)$ surface at 14 positions, with the lattice held fixed at its $f c c$ equilibrium positions. Au atoms in the surface layer are indicated as closed black circles. The blue solid circle indicates the sub-surface Au atom below the hcp hollow site (7). The yellow solid circles indicate the subsurface Au atom below the $f c c$ hollow site (10). Positions 110 shown in a) are lines of lattice symmetry. Positions 11-14 b) are non-symmetric. The [101] direction is along a line connecting positions 5 and 10. The [112] direction is along a line connecting points 1 and 10 . A top view of the $f c c$ lattice is shown in c). A primitive (Wigner-Seitz) cell is defined in d).

and a substrate, respectively, has the following form:

$$
\begin{aligned}
E= & \sum_{i} E_{\mathrm{Au}}^{(\mathrm{c})}\left(n_{\mathrm{i}}\right)+E_{\mathrm{H}}^{(\mathrm{c})}\left(n_{\mathrm{H}}\right)+\frac{1}{2} \sum_{i, j} V_{\mathrm{Au}}\left(r_{i j}\right) \\
& +\frac{1}{2} \sum_{i}\left[V_{\mathrm{H}, \mathrm{Au}}\left(r_{\mathrm{H} j}\right)+V_{\mathrm{Au}, \mathrm{H}}\left(r_{\mathrm{H} j}\right)\right]-V_{\mathrm{ref}}
\end{aligned}
$$

where the index $\mathrm{H}$ refers to the hydrogen atom and index $i$ runs over Au atoms; $r_{i j}$ is a distance between gold atoms labeled by indices $i$ and $j ; r_{\mathrm{H} i}$ is a distance between a hydrogen and a gold atom $i$. The cohesive functions $E_{\mathrm{Au}}^{(\mathrm{c})}$ and $E_{\mathrm{H}}^{(\mathrm{c})}$ give the energy of a corresponding atom in the reference system. Their dependence on the embedding 
density is parameterized in terms of neutral sphere radii $s_{i}$ of gold atoms and $s_{\mathrm{H}}$ of hydrogen by following expressions

$$
\begin{aligned}
& E_{\mathrm{Au}}^{(\mathrm{c})}\left(n_{\mathrm{i}}\right)=E_{0, \mathrm{Au}}\left[1+\lambda_{\mathrm{Au}}\left(s_{i}-s_{0, \mathrm{Au}}\right)\right] \mathrm{e}^{-\lambda_{\mathrm{Au}}\left(s_{i}-s_{0, \mathrm{Au}}\right)}-E_{0, \mathrm{Au}}, \\
& E_{\mathrm{H}}^{(\mathrm{c})}\left(n_{\mathrm{H}}\right)=E_{0, \mathrm{H}}\left[1+\lambda_{\mathrm{H}}\left(s_{\mathrm{H}}-s_{0, \mathrm{H}}\right)\right] \mathrm{e}^{-\lambda_{\mathrm{H}}\left(s_{\mathrm{H}}-s_{0, \mathrm{H}}\right)}
\end{aligned}
$$

The relation between a neutral sphere radius $s$ and the embedding density $n$ is modeled by exponential $n=n_{0} \exp \left[-\eta\left(s_{\mathrm{H}}-s_{0, \mathrm{H}}\right)\right]$, which allows to determine $s$ as function of atomic positions:

$$
\begin{aligned}
s_{i} & =-\frac{\ln \left[\left(\sigma_{i}+\chi_{\mathrm{Au}, \mathrm{H}} \sigma_{i, \mathrm{H}}\right) / 12\right]}{\beta \eta_{2, \mathrm{Au}}}, \\
s_{\mathrm{H}} & =-\frac{\ln \left[\chi_{\mathrm{H}, \mathrm{Au}} \sigma_{\mathrm{H}, \mathrm{Au}} / 12\right]}{\beta \eta_{2, \mathrm{H}}}
\end{aligned}
$$

with geometric factor $\beta=\sqrt[3]{\frac{16 \pi}{3 \sqrt{2}}}$, and

$$
\begin{aligned}
& \sigma_{i}=\gamma_{1, \mathrm{Au}}^{-1} \sum_{j} \mathrm{e}^{-\eta_{2, \mathrm{Au}}\left(r_{i j}-\beta s_{0, \mathrm{Au}}\right)} \theta\left(r_{i j}\right), \\
& \sigma_{i, \mathrm{H}}=\gamma_{1, \mathrm{Au}}^{-1} \mathrm{e}^{-\eta_{2, \mathrm{H}}\left(r_{\mathrm{H} i}-\beta s_{0, \mathrm{H}}\right)} \theta\left(r_{\mathrm{H} i}\right), \\
& \sigma_{\mathrm{H}, \mathrm{Au}}=\gamma_{1, \mathrm{H}}^{-1} \sum_{i} \mathrm{e}^{-\eta_{2, \mathrm{Au}}\left(r_{\mathrm{H} i}-\beta s_{0, \mathrm{Au}}\right)} \theta\left(r_{\mathrm{H} i}\right) .
\end{aligned}
$$

Here the coefficients $\chi_{\mathrm{Au}, \mathrm{H}}$ and $\chi_{\mathrm{H}, \mathrm{Au}}$ are given by

$$
\chi_{\mathrm{Au}, \mathrm{H}}=\frac{n_{0, \mathrm{H}}}{n_{0, \mathrm{Au}}} \mathrm{e}^{-\eta_{1}\left(s_{0, \mathrm{H}}-s_{0, \mathrm{Au}}\right)}, \quad \chi_{\mathrm{H}, \mathrm{Au}}=\chi_{\mathrm{Au}, \mathrm{H}}^{-1}
$$

and the function

$$
\theta(r)=\left(1+\mathrm{e}^{\alpha\left(r-r_{\mathrm{c}}\right)}\right)^{-1}
$$

serves as a smooth cut-off, where $\alpha=\ln 10^{5} /\left(r_{\mathrm{r}}-r_{\mathrm{c}}\right)$ and $r_{\mathrm{r}}=4 r_{\mathrm{c}} /(\sqrt{3}+2)$. The cutoff radius $r_{\mathrm{c}}=a_{0} \sqrt{1.5}$ is taken to be the distance to the next-next-nearest neighbors of the $f c c$ lattice with the lattice constant $a_{0}$.

Another contribution to the total energy Eq. (1) comes from the correction term accounting for the difference between the reference system and the real system. It is represented as pairwise interaction energies of the form

$$
\begin{aligned}
& V_{\mathrm{Au}}\left(r_{i j}\right)=-V_{0, \mathrm{Au}} \mathrm{e}^{-\kappa_{\mathrm{Au}}\left(\beta^{-1} r_{i j}-s_{0, \mathrm{Au}}\right)} \theta\left(r_{i j}\right) \gamma_{2, \mathrm{Au}}^{-1}, \\
& V_{\mathrm{Au}, \mathrm{H}}\left(r_{\mathrm{H} i}\right)=-\chi_{\mathrm{Au}, \mathrm{H}} V_{0, \mathrm{Au}} \mathrm{e}^{-\kappa_{\mathrm{H}}\left(\beta^{-1} r_{\mathrm{H} i}-s_{0, \mathrm{H}}\right)} \theta\left(r_{\mathrm{H} i}\right) \gamma_{2, \mathrm{Au}}^{-1}, \\
& V_{\mathrm{H}, \mathrm{Au}}\left(r_{\mathrm{H} i}\right)=-\chi_{\mathrm{H}, \mathrm{Au}} V_{0, \mathrm{H}} \mathrm{e}^{-\kappa_{\mathrm{Au}}\left(\beta^{-1} r_{\mathrm{H} i}-s_{0, \mathrm{Au}}\right)} \theta\left(r_{\mathrm{H} i}\right) \gamma_{2, \mathrm{H}}^{-1}, \\
& V_{\mathrm{ref}}=-6 V_{0, \mathrm{Au}} \sum_{i} \mathrm{e}^{-\kappa_{\mathrm{Au}} s_{i}}-6 V_{0, \mathrm{H}} \mathrm{e}^{-\kappa_{\mathrm{H}} s_{\mathrm{H}}}
\end{aligned}
$$


The values of $\gamma_{1}$ and $\gamma_{2}$ that enter Eqs. (5) and (8) as weighting parameters are defined by the sums running over the nearest $(n=1)$, next-nearest $(n=2)$ and next-nearest-next $(n=3)$ neighbor atoms:

$$
\begin{aligned}
& \gamma_{1, \alpha}=\sum_{n=1}^{3} x_{\alpha, n} \mathrm{e}^{-\eta_{2, \alpha}\left(r_{\alpha, n}-\beta s_{0, \alpha}\right)}, \\
& \gamma_{2, \alpha}=\sum_{n=1}^{3} x_{\alpha, n} \mathrm{e}^{-\kappa_{\alpha}\left(\beta^{-1} r_{\alpha, n}-s_{0, \alpha}\right)},
\end{aligned}
$$

where index $\alpha$ labels a type of atoms $(\alpha=\mathrm{H}, \mathrm{Au})$ and

$$
x_{\alpha, n}=\frac{b_{n}}{12\left(1+\mathrm{e}^{\alpha\left(r_{\alpha, n}-r_{\mathrm{c}}\right)}\right)}
$$

with $b_{1}=12, b_{2}=6$, and $b_{3}=24$, since there are 12 nearest neighbor atoms, 6 nextnearest neighbors and 24 next-next-nearest neighbor atoms, respectively. For the $f c c$ lattice the distance to the neighbors is defined by the relation $r_{\alpha, n}=\beta s_{0, \alpha} \sqrt{n}$.

When performing the fit, the gold atom positions of the reference system were taken as those of a perfect $f c c$ seven-layer slab with 90 atoms per layer and a lattice constant $a_{0}=4.201 \AA$. The coordinates of the $\mathrm{H}$-atom were identical to those used in the DFT calculations described above in the discussion concerning Figs. 1 and 2.

The fitting procedure ends up with the following parameters: $\eta_{2, \mathrm{H}}, s_{0, \mathrm{H}}, n_{0, \mathrm{H}}, \lambda_{\mathrm{H}}$, $\kappa_{\mathrm{H}}, V_{0, \mathrm{H}}, E_{0, \mathrm{H}}$ for the hydrogen adsorbate and $\eta_{2, \mathrm{Au}}, s_{0, \mathrm{Au}}, n_{0, \mathrm{Au}}, \lambda_{\mathrm{Au}}, \kappa_{\mathrm{Au}}, V_{0, \mathrm{Au}}, E_{0, \mathrm{Au}}$ for the gold lattice. Those have to be determined during the fit. We performed the fit with the standard fitting tools implemented into Mathematica 8 (Levenberg-Marquadt algorithm).

\subsection{Molecular dynamics simulations}

For the all the MD simulations involving the EMT potential, we used the ASEpackage [49]. This package was specifically designed to simulate atomic (or molecular) scattering from surfaces and contains a built-in EMT-potential [23]. We replaced the standard values of EMT-potential parameters implemented in the original package with those found by us from the fit described above. Within this environment, we used the Verlet-algorithm to calculate 100000 trajectories for five different incidence conditions (see Table 1 and Fig. 1c). We performed calculations on a $5 \times 5 \times 7$ gold slab, with the lower layer held fixed, for $E_{\mathrm{i}}=5 \mathrm{eV}$ and $T_{\mathrm{S}}=10 \mathrm{~K}$. $T_{\mathrm{S}}$ is set by sampling the initial velocities from a Maxwell-Boltzmann distribution.

The trajectories were started with the H-Atom at $z=6 \AA$ above the surface with randomly chosen $x$-y-positions. We allowed a maximum propagation time of $10 \mathrm{ps}$ and a time step of $0.1 \mathrm{fs}$. As soon as the $\mathrm{H}$-atom again reached a distance of $z>6 \AA$, the trajectory was halted. We denote those trajectories as scattered. A fraction $(\sim 10 \%)$ of the trajectories went completely through the slab or had not left the slab after $10 \mathrm{ps}$. These trajectories are regarded as trapped We also distinguish between $\mathrm{H}$-atoms that scatter from the surface - referred to as scattering without penetration - and trajectories that 


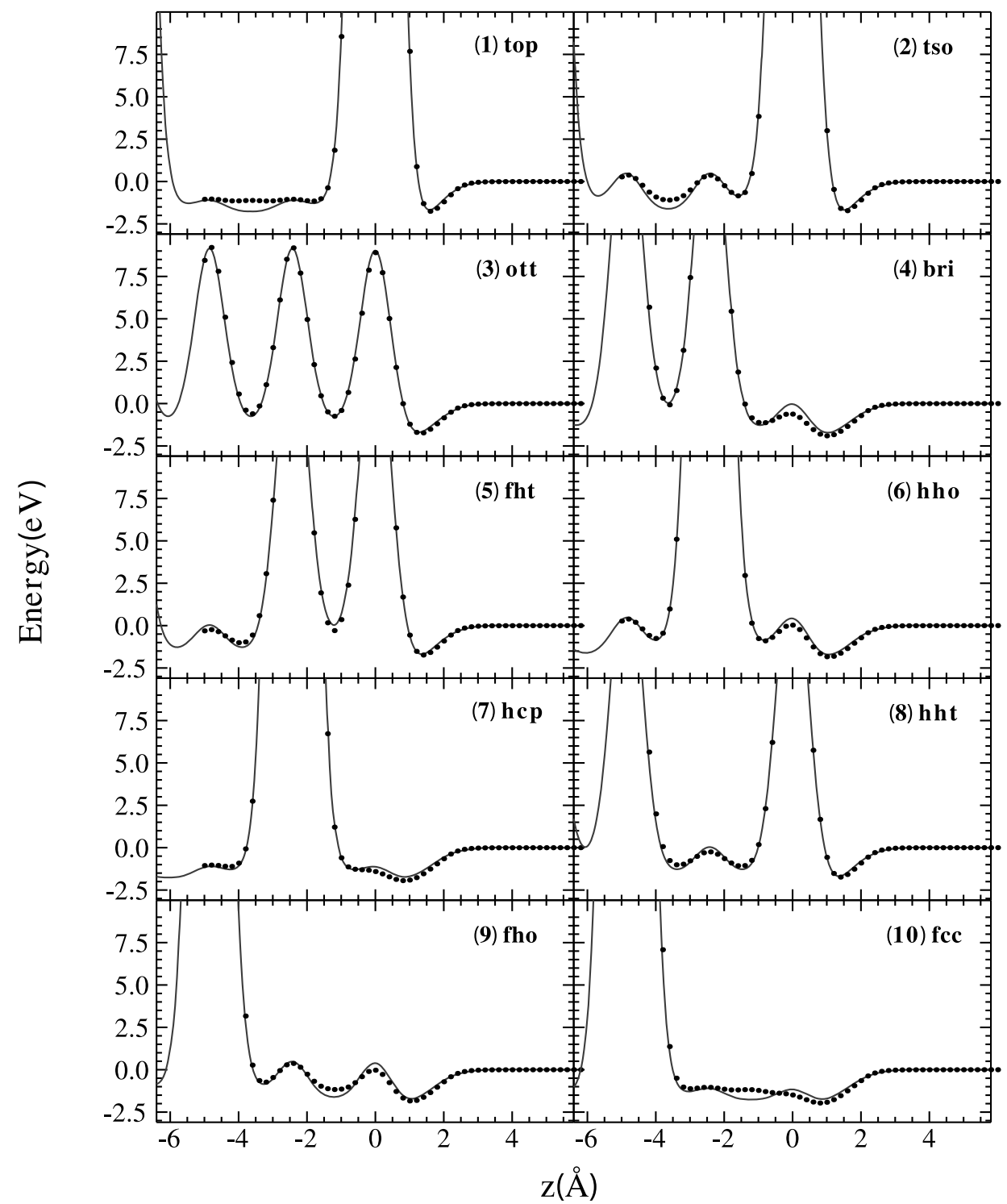

Fig. 2. DFT energies (black filled circles) and EMT fit (solid line). Each plot is along a line normal to the (111) surface at a position indicated by Fig. 1a.

penetrate (achieve $z<0$ ) before re-emerging - referred to as scattering with penetration. In the AIMD calculations, the Au lattice was modeled with VASP using a 4-layer, $2 \times 2$ slab in the same way as described in Sect. 2.1. To check that we do not get any influence due to $\mathrm{H}$-image interaction we compared the energies calculated with the $3 \times 3$ cell to those of the $2 \times 2$ cell with the hydrogen atom for several selected $\mathrm{H}$-atom positions inside of and on the slab, and for both cells the energies relative to their reference 
Table 1. Scattering and penetrating probabilities obtained from MD simulations.

\begin{tabular}{lllllll}
\hline $\begin{array}{l}\text { Incidence } \\
\text { case }\end{array}$ & $\theta_{\text {inc }}\left(^{\circ}\right)$ & $\begin{array}{l}\text { Azimuthal } \\
\text { direction }\end{array}$ & $P_{\mathrm{sc}}^{\mathrm{a}}$ & $P_{\mathrm{np}}^{\mathrm{b}}$ & $P_{\mathrm{lost}}{ }^{\mathrm{c}}$ & $\left(1-\frac{P_{\mathrm{np}}}{P_{\mathrm{sc}}}\right) \mathrm{d}$ \\
\hline $\mathrm{A}$ & 0 & - & 0.82 & 0.39 & 0.18 & 0.53 \\
$\mathrm{~B}$ & 15 & {$[10 \overline{1}]$} & 0.80 & 0.39 & 0.20 & 0.51 \\
$\mathrm{C}$ & 15 & {$[11 \overline{2}]$} & 0.79 & 0.40 & 0.21 & 0.50 \\
$\mathrm{D}$ & 60 & {$[10 \overline{1}]$} & 0.90 & 0.70 & 0.10 & 0.23 \\
$\mathrm{E}$ & 60 & {$[11 \overline{2}]$} & 0.88 & 0.64 & 0.12 & 0.27 \\
\hline
\end{tabular}

\footnotetext{
a Trajectories that are found more than $6 \AA$ from the surface $(z>6 \AA)$ after $10 \mathrm{ps}$.

${ }^{\mathrm{b}}$ Trajectories that scatter from surface sites by single, double or multi-bounce. This includes trajectories that scattering from hollow sites that may have brief periods with $z<0$. ${ }^{c}$ Trajectories that have not re-emerged from the slab or have been transmitted through the slab after $10 \mathrm{ps}$. This may be considered as the trapping probability within the electronically adiabatic approximation.

${ }^{\mathrm{d}}$ The fraction of scattering trajectories that penetrate the solid.
}

energies converge to similar values within $0.02 \mathrm{eV}$ difference. Therefore, we conclude that a $2 \times 2$ cell is adequate. Only the upper 3 layers of the slab were allowed to move. Molecular dynamics simulations were performed using the Verlet algorithm [50,51] with a time-step of $0.1 \mathrm{fs}$ and a surface temperature of $120 \mathrm{~K}$. The implementation of the AIMD used here is mostly the same as the one employed earlier [20] in calculations on $\mathrm{D}_{2}+\mathrm{Cu}(111)$. An important feature of the present calculations on $\mathrm{H}+\mathrm{Au}(111)$ is that spin-polarized DFT was used to model the presence of (partial) spin on $\mathrm{H}$ when it is far away from the surface. A more complete description of the AIMD calculations will be presented in a future paper [52]. We used the results from the AIMD calculations to check the quality of our fit.

\section{Results}

The results section is organized into four parts. In Sect. 3.1 we present all information necessary to implement the new PES and characterize the quality of the EMT fit to the DFT data. We also provide a qualitative description of the $\mathrm{H} / \mathrm{M}$ interaction. In Sect. 3.2 we examine the question of how well the EMT PES describes the displacements of Au atoms from the equilibrium positions of a bulk lattice. In Sect. 3.3 we evaluate the quality of the PES by comparison to DFT calculations. In Sect. 3.4 we present preliminary MD calculations using the new PES.

\subsection{Presentation of the new potential energy surface}

The EMT parameters used to fit the DFT data are presented in Table 2. The parameters for gold [23] and two parameters for hydrogen [1] were held fixed during the optimization procedure. Figure 2 shows the complete graphical comparison of the EMT fit (solid line) to the DFT data (points). This figure shows the $z$-dependence of the potential energy over 10 symmetry sites of the unit cell described (see Sect. 2.1 and Fig. 1a). The 
Table 2. EMT parameters used to fit the DFT data.

\begin{tabular}{lcc}
\hline EMT constants & $\mathrm{H}$ & $\mathrm{Au}$ [23] \\
\hline$s_{0}(\mathrm{bohr})$ & 0.470 & 3.00 \\
$\eta_{2}\left(\mathrm{bohr}^{-1}\right)$ & 4.878 & 1.674 \\
$\lambda\left(\mathrm{bohr}^{-1}\right)$ & 7.906 & 2.182 \\
$\kappa\left(\mathrm{bohr}^{-1}\right)$ & 4.541 & 2.873 \\
$V_{0}\left(\mathrm{bohr}^{-1}\right)$ & 0.298 & 2.321 \\
$n_{0}\left(\mathrm{bohr}^{-3}\right)$ & $0.027[1]$ & 0.00703 \\
$E_{0}(\mathrm{eV})$ & $-2.371[1]$ & -3.8 \\
\hline
\end{tabular}

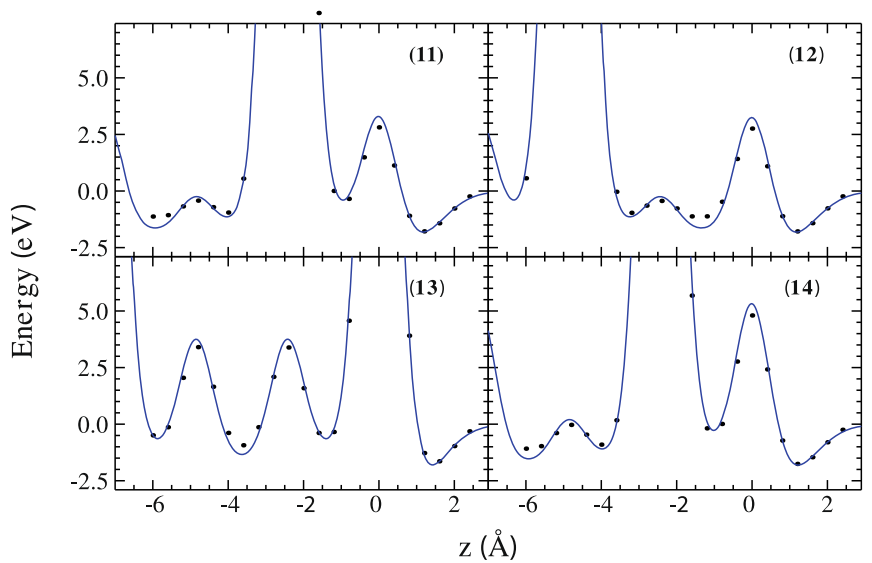

Fig. 3. DFT energies (solid black circles) and EMT fit (solid blue line). Each plot is along a line normal to the (111) surface at a position indicated by Fig. $1 \mathrm{~b}$.

standard error to the fit is $121 \mathrm{meV}$, similar to the errors expected from DFT/GGA calculations due to the exact form of the exchange correlation functional being unknown. We carried out additional DFT calculations along linear grids normal to the surface at four non-symmetry sites within the unit cell. Note that these DFT energies were not used in the optimization procedure. These sites (labeled 11-14) are also shown in Fig. 1b. A graphical comparison of our EMT PES to these DFT energies is shown in Fig. 3. The overall standard error for all four sites is $194 \mathrm{meV}$.

The biggest deficiency of the PES visible in Fig. 2 is the description of the energies in sub-surface regions where the $\mathrm{H}$-atom is furthest from the nearest Au atom. This is apparent in the subsurface sites along the top, tso (top site one-third-to-bridge-site), fho ( $f c c$ hollow, one-third-on-(top- $f c c-h c p$ )-angle-bisector), and $f c c$ positions ( $c f$. Fig. 1 , sites 1,2, 9 and 10, respectively). The largest deviation between the EMT fit and the DFT points is $0.736 \mathrm{eV}$ in the large subsurface hollow below the top surface site. Note that the subsurface sites along the hcp position are more accurately reproduced. This may reflect inaccuracies in the EMT description of the electron density at long distances from each $\mathrm{Au}$ atom. This issue deserves further analysis in future work. 


\subsection{Evaluation of the model of $\mathbf{H}$-atom-to-lattice coupling}

To examine how well the new PES describes Au lattice relaxation in the presence of an $\mathrm{H}$-atom, we first compared relaxed geometries (i.e. energetic extrema) found by DFT to those from the new PES. We made such comparisons for $20 \mathrm{H}$-atom positions corresponding to local extrema of PES above as well as below the surface. The lattice relaxation energy obtained from DFT varies between -0.02 and $-0.26 \mathrm{eV}$.

Lattice relaxation energies from the EMT-based PES are similar in magnitude to those obtained from DFT; the largest relaxation energy being less than $0.8 \mathrm{eV}$. It is not clear that lattice relaxation in the presence of an $\mathrm{H}$ atom is described by the present PES with high accuracy in the regions of configuration space close to the overall energetic minima. On the other hand, for application of MD presented in this paper - scattering of high incidence energy $\mathrm{H}$-atom with low surface temperature Au solid - full lattice relaxation to energetic minima is unimportant, since the $\mathrm{H}$ atom moves much more rapidly than the $\mathrm{Au}$ atoms and the thermal deviations from the $f c c$ lattice structure are minimal. We thus assert that the results of our MD calculations under the conditions used in this paper are accurate enough to learn about the adiabatic scattering dynamics. This, of course, means that the present results, which are semi-empirical, should be tested in the future against full first principles methods.

In order to characterize how well our EMT-based PES reproduces H-atom interactions with the solid for non- $f c c$ lattice structures relevant to these applications, we used geometries from thirteen AIMD trajectories $(2 \times 2 \times 4$ supercell size $)$ to calculate EMT-based energies and compared these with the AIMD energies for every time step of the trajectories. A representative comparison of AIMD and EMT energies along one AIMD trajectory is shown in Fig. 4. One notices that at $t=0$, there is a $\sim 0.1 \mathrm{eV}$ energy difference between the DFT energies coming from AIMD calculations and the energies of the EMT-based PES as implemented in ASE. This is solely due to differences in how the two methods describe lattice distortion away from the $f c c$ structure, since the $\mathrm{H}$ atom is still so far from the solid at $t=0$. It is important to understand that this error is approximately constant during all of our AIMD trajectories, since the time scale of $\mathrm{Au}$ atom motion is much longer than that of $\mathrm{H}$ atom motion; from the Debye temperature of $\mathrm{Au}$ [53], the frequency of gold atom motion can be estimated to have a period of about $260 \mathrm{fs}$. Hence we are justified in offsetting the EMT energies with respect to the AIMD energies by the $t=0$ energy difference. When we do this for all of the AIMD based DFT data, we obtain a histogram of the deviations, which is also shown in Fig. 4 (lower panel). These deviations account for the difference in $\mathrm{H}$-atom to lattice coupling energies over the time scales of relevance to the scattering, typically less than $1000 \mathrm{fs}$. Although there are some points of comparison with large deviations, one can see that the vast majority of deviations lie between $\pm 0.1 \mathrm{eV}$ and the distribution of deviations peaks near zero. This leads us to conclude that even this preliminary PES is suitable for simulations of high energy $\mathrm{H}$-atom scattering at low surface temperature. Despite the reasonableness of these arguments, we wish to emphasize that Au lattice force field implemented in this work remains approximate since it is based on a semi-empirical approach. The present calculations are no substitute for first principles calculations that we hope to see in the future. 

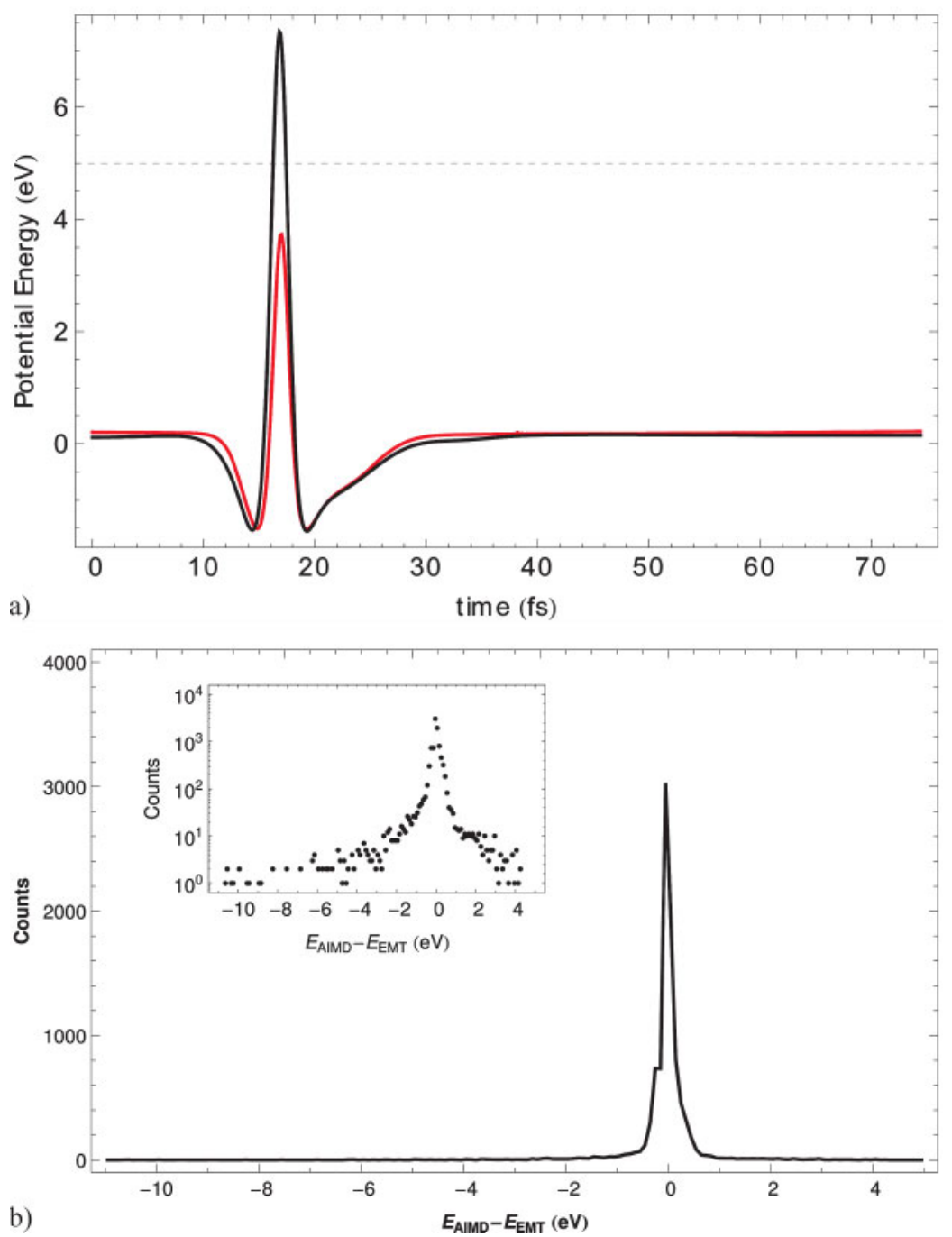

Fig. 4. The EMT fit for structures relevant to scattering. a) DFT potential energies calculated for an AIMD trajectory exhibiting a single bounce at the surface (red solid line) is compared to the EMT energies at the same coordinates (black solid line). b) A histogram of the energy difference between AIMD and EMT for all DFT data in the 13 AIMD trajectories with an inset in logarithmic scale. The incidence energy is shown as a dashed line.

\subsection{Comparison of selected energy points with previous work}

There have been only a few calculations of the H/Au interaction, the most recent of which is Ref. [2] (see Table 3). Our PES predicts that the $f c c$ and $h c p$ hollow sites (sites 10 and 7 respectively in Fig. 1a) represent the most favorable adsorption sites on the 
Table 3. Comparison between adsorption sites and energies for EMT-PES and DFT results.

\begin{tabular}{|c|c|c|c|c|c|c|}
\hline & \multicolumn{2}{|c|}{ EMT-PES } & \multicolumn{2}{|c|}{ R48PBE-DFT } & \multicolumn{2}{|c|}{ PW91-DFT [2] } \\
\hline & Site & Energy $(\mathrm{eV})$ & Site & Energy $(\mathrm{eV})$ & Site & Energy $(\mathrm{eV})$ \\
\hline On the surface & $f c c / h c p$ & -1.84 & $f c c$ & -1.97 & $f c c$ & 2.18 \\
\hline $\begin{array}{l}1^{\text {st }} \text { interlayer } \\
(0.0 \text { to }-2.4 \AA)\end{array}$ & TUT $^{\mathrm{a}} /$ Octa $^{\mathrm{b}}$ & -1.34 & Octa & -1.19 & TUT & -1.55 \\
\hline $\begin{array}{l}2^{\text {nd }} \text { interlayer } \\
(-2.4 \text { to }-4.8 \AA)\end{array}$ & Octa & -1.85 & Octa & -1.15 & TUT & -1.45 \\
\hline
\end{tabular}

a Tetrahedral under top,

${ }^{\mathrm{b}}$ Octahedral.

surface and that these have nearly equal energies. These energy values deviate slightly from the ones we obtained using the R48PBE functional. The latter values compare well to the findings of Ferrin $e t a l$. who predict a preference for the $f c c$ site. The binding energy of $-2.18 \mathrm{eV}$ found by Ferrin et al. is somewhat larger than the $-1.97 \mathrm{eV}$ found here (Table 3). This discrepancy most likely reflects differences in the DFT energies for the R48PBE functional used in this work and the PW91 function used in Ref. [2]. The PW91 functional is known to overestimate adsorption energies [54].

In the first subsurface layer, extending from 0.0 to $-2.4 \AA$, we find that the octahedral site (Octa, $c f$. Fig. 2, site 10) of our PES is significantly more stable $(-1.85 \mathrm{eV})$ than the value of $-1.19 \mathrm{eV}$ we obtained from SPR-DFT calculations. Ferrin et al. found a preference for the TUT site and a binding energy of $-1.55 \mathrm{eV}$. Our DFT-calculations found an energy of $-1.15 \mathrm{eV}$ for the TUT site whereas the EMT-PES energy amounts to $-1.34 \mathrm{eV}$ here.

In the second subsurface layer, extending between -2.4 and $-4.8 \AA$, we observe a distinct preference for the octahedral site ( $c f$. Fig. 1, site 1) which also forms the overall minimum of the PES with a binding energy of $-1.85 \mathrm{eV}$, which is in direct contradiction to the calculations reported by Ferrin et al. who find that adsorption on the surface is favorable compared to absorption into the surface and predict a preference for the TUT site. However, also our DFT data predicts a slight preference of the octahedral site compared to the TUT site, even if our EMT-based PES overestimates the depth of this minimum (Table 3). Moreover, Ferrin et al. [2] also mention that for most other $f c c$ metals, including $\mathrm{Cu}(111)$, the absorption to the octahedral sites is favored over absorption to the TUT sites.

The above comparison indicates that the deviations of the fit from the R48PBE-DFT values are in the same energy region as the differences between the energy calculated from the R48PBE- and the PW91-functionals.

In a previous study, EMT was fit to DFT data for $\mathrm{H}$ on Copper with a root mean square error of $0.2 \mathrm{eV}$ [1]. The present fitâs root mean square error of $0.18 \mathrm{eV}$ is somewhat better. The present EMT based PES is clearly most accurate for surface binding sites, mainly due to the fact that the EMT fit to the DFT exhibits its largest errors for subsurface avities, where the distance to the nearest $\mathrm{Au}$ atom is largest. Due to this consideration and due to the fact that lattice relaxation in the presence of $\mathrm{H}$-atom is only approximately described by this PES we restrict our analysis of MD calculations to 
non-penetrating collisions with high incidence energy, $E_{\mathrm{i}}=5 \mathrm{eV}$, at low surface temperature, $T_{\mathrm{S}}=10 \mathrm{~K}$.

\subsection{Molecular dynamics calculations}

A key advantage of the fitted EMT-based PES developed in this work is the possibility to produce hundreds of thousands of MD trajectories in a reasonable time, even on a moderate size computational cluster, thus, making feasible the detailed theoretical study of the angular and energy resolved features of the scattering. In this section we describe such calculations.

Specifically, we have simulated energy and angle resolved scattering for five incidence polar and azimuthal angles (Table 1). As we shall see below, we are able to identify several distinctly different scattering mechanisms. These include: 1) trajectories that penetrate the surface and 2) trajectories that scatter from surface sites (surface scattering). The surface scattering can also be divided into at least three categories. We see two different types of scattering from a top sites, one occurring with a single bounce and one leading predominantly to two bounce events. We also discern a second single bounce scattering mechanism, which transfers energy to the solid more efficiently and is associated with impact at or near hollow sites. Finally, we can identify near-parallel to surface back-scattering events. These are the least efficient of all in transferring $\mathrm{H}$-atom incidence energy to Au atom motion.

Figure 5 shows the total translational energy loss distribution (black dot-dashed curves) for five incidence conditions varying from normal incidence to $60^{\circ}$ from normal for two incidence azimuths: namely along the $10 \overline{1}$ and $11 \overline{2}$ directions. See captions for Figs. 5 and 1 for details. These distributions all peak at about $\Delta E_{\text {loss }} \sim 150 \mathrm{meV}$ and slowly diminish in probability with larger energy loss. The blue thick solid lines in Fig. 5 show non-penetrating collisions. The difference between the black and blue lines represents trajectories that penetrate the solid. Such penetrating trajectories are nearly absent in panels d) and e) where $\theta_{\mathrm{i}}=60^{\circ}$ is the largest. Table 1 also shows the penetrating probability for all of the incidence conditions of this work. We do not know at present if - or to what extent - penetrating collisions re-emerge from an actual gold surface, since weak non-adiabatic effects might be important for such trajectories. One important conclusion we can immediately draw based on Fig. 5, is that penetrating collisions are predicted to be less important at larger incidence angles.

The red solid curves in Fig. 5 show the contribution of single-bounce scattering processes. Here, we define a bounce as an event where the $\mathrm{H}$-atom enters the strong interaction region, and the change in the force exerted on the $\mathrm{H}$-atom exceeds a certain threshold $\left(0.1 \frac{\mathrm{amu} \cdot \AA}{\mathrm{fs}^{2}} \approx 10.4 \mathrm{eV} / \AA\right)$. An important and simple observation here is that single bounce scattering makes up a large fraction of the overall trajectories regardless of the chosen incidence angles. The single-bounce energy loss distributions are in general quite narrow, peaking between $\Delta E_{\text {loss }}=100$ and $150 \mathrm{meV}$. It is interesting to note that this is close to the attractive Baule limit $(140 \mathrm{meV})$; that is, the energy loss expected from a zero-impact parameter, binary collision between an $\mathrm{H}$ - and an Au-atom, with prior acceleration of the $\mathrm{H}$-atom due to the $2 \mathrm{eV}$ attractive binding energy.

Also shown in Fig. 5 is the contribution of multiple bounce collisions (dashed red curve) as well as double bounce trajectories (dotted green curve). We note that the im- 
a)

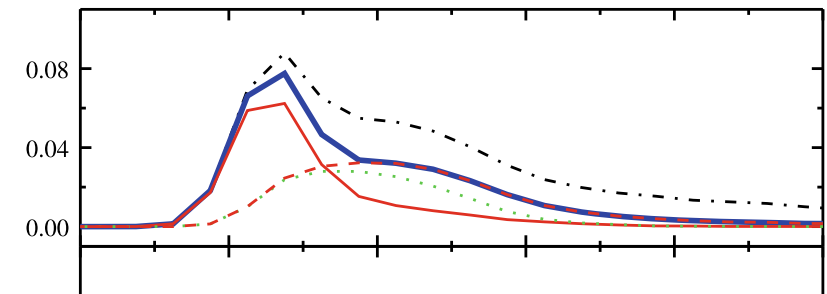

b)

c)

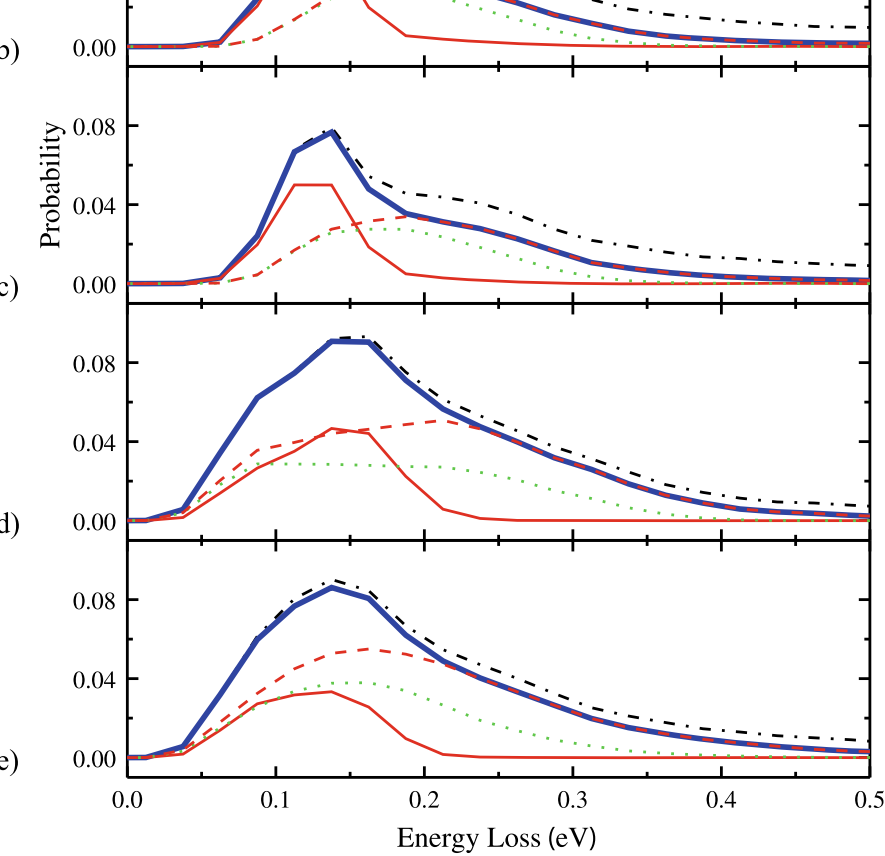

Fig. 5. Total angle integrated $\mathrm{H}$-atom energy loss distributions. Five incidence conditions are shown. (a) $\theta_{\mathrm{i}}=0^{\circ} ;$ (b) $\theta_{\mathrm{i}}=15^{\circ}, n_{\mathrm{i}}=[10 \overline{1}]$; (c) $\theta_{\mathrm{i}}=15^{\circ}, n_{\mathrm{i}}=[11 \overline{2}]$; (d) $\theta_{\mathrm{i}}=60^{\circ}, n_{\mathrm{i}}=[10 \overline{1}]$; (e) $\theta_{\mathrm{i}}=60^{\circ}, n_{\mathrm{i}}=[11 \overline{2}]$. Black dot-dashed - total scattered, blue thick solid - non-penetrating, red solid - single-bounce nonpenetrating. Green dotted - double-bounce. Dashed - multiple bounce. Binning interval is $2.5 \mathrm{meV}$.

portance of double bounce collisions is only weakly dependent of incidence conditions, gaining slowly in importance at higher incidence angles in comparison to single bounce collisions. Second, the fraction of multiple bounce collisions that occur with only two bounces is highest at normal incidence, where there are essentially no triples or higher. The energy loss due to double bounce trajectories is consistent with a value twice the attractive Baule limit. The importance of triples and higher slowly increases with higher incidence angles.

Close inspection of Fig. 5 reveals that for $\theta_{\mathrm{i}}=60^{\circ}$, the single bounce trajectories are made up of two components, one peaking at $\Delta E_{\text {loss }} \sim 75 \mathrm{meV}$ and one peaking at 

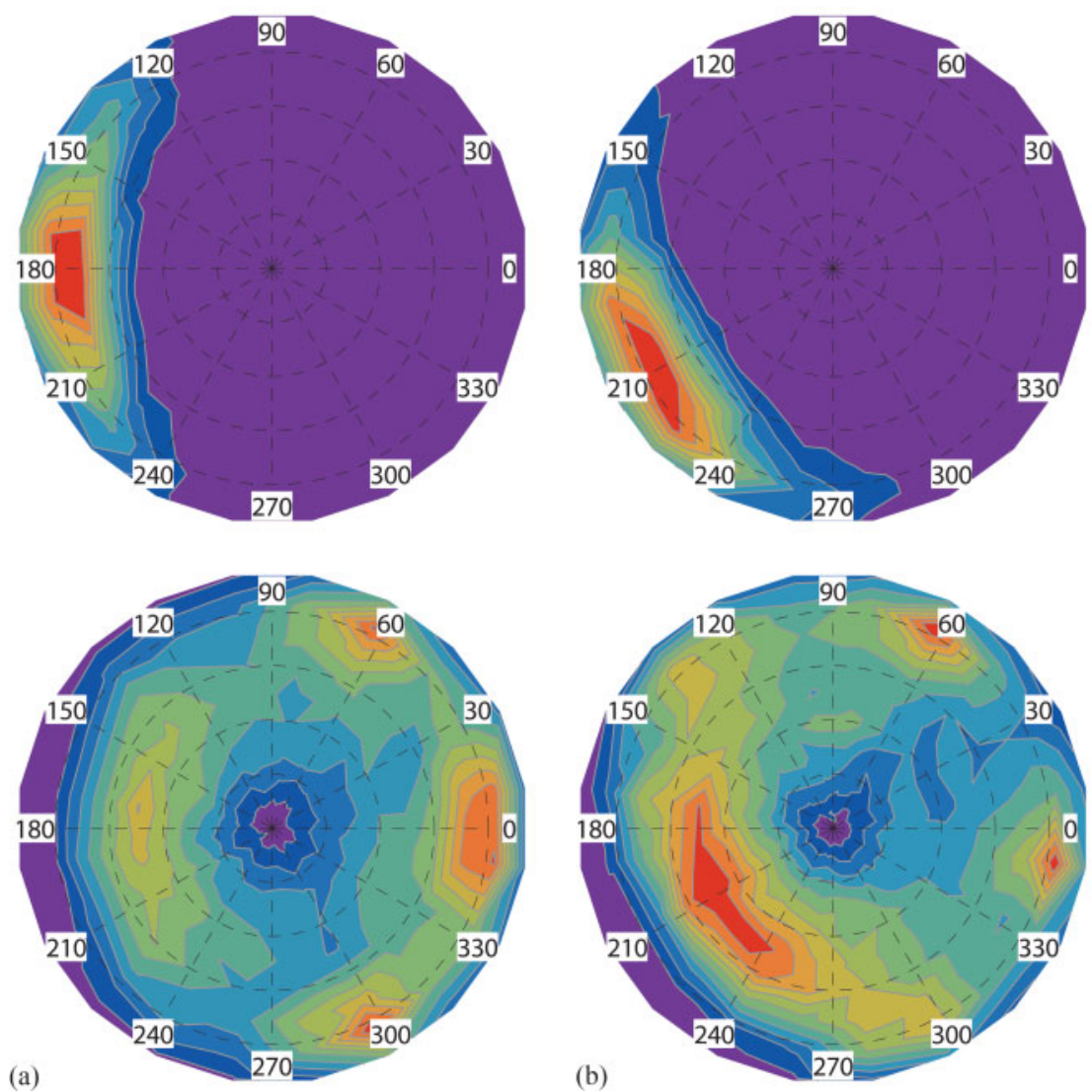

Fig. 6. Energy resolved angular distributions for single bounce events. a) $\theta_{\mathrm{i}}=60^{\circ}, n_{\mathrm{i}}=[10 \overline{1}]$; b) $\theta_{\mathrm{i}}=60^{\circ}$, $n_{\mathrm{i}}=[11 \overline{2}]$. The polar scattering angle, $\theta_{\mathrm{s}}$, is denoted by the distance from the center of the plots. The distance between each dashed ring represents $\Delta \theta_{\mathrm{s}}=20^{\circ}$. The azimuthal scattering angle, $\phi_{\mathrm{s}}$, is indicated explicitly. The azimuthal incidence angle is indicated by the arrows and by the crystallographic notation. Purple is minimum and red is maximum.

$\Delta E_{\text {loss }} \sim 130 \mathrm{meV}$. Figure 6 shows energy resolved angular distributions of the single bounce events for two incidence conditions with $\theta_{\mathrm{i}}=60^{\circ}$. This confirms the presence of at least two types of single bounce trajectories. Outgoing single bounce trajectories with $\Delta E_{\text {loss }}<100 \mathrm{meV}$ travel nearly parallel to the surface, their scattering angular distributions peaking at $\theta_{\mathrm{s}}=75^{\circ}$, and are scattered in the backward direction. For outgoing single bounce trajectories with $100<\Delta E_{\text {loss }}<200 \mathrm{meV}$, the situation is more complex. Here, the scattering angle peaks near $\theta_{\mathrm{s}}=50^{\circ}$ and is again predominantly back scattered for [112] scattering, but for scattering incident along the [101] direction, forward scattering is also seen. In both cases a significant contribution of sideways scattering is seen still appearing in the forward hemisphere. We may also use MD to calculate angle resolved energy loss distributions. Figure 7 shows energy loss distributions found for scattering conditions: $\theta_{\mathrm{i}}=60^{\circ}, n_{\mathrm{i}}=[10 \overline{1}], \theta_{\mathrm{s}}=75^{\circ}, \phi_{\mathrm{s}}=180^{\circ}$ (Fig. 7a) 


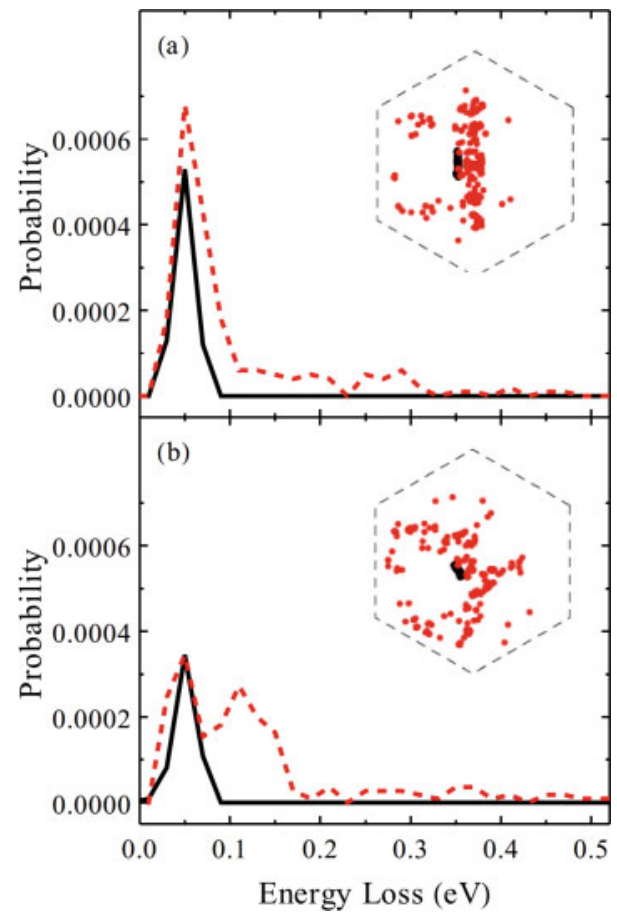

Fig. 7. Nearly elastic adiabatic scattering of $\mathrm{H}$ atoms from $\mathrm{Au}(111)$. (a) $\theta_{\mathrm{i}}=60^{\circ}, n_{\mathrm{i}}=[10 \overline{1}], \theta_{\mathrm{s}}=75^{\circ}$, $\phi_{\mathrm{s}}=180^{\circ}$. (b) $\theta_{\mathrm{i}}=60^{\circ}, n_{\mathrm{i}}=[11 \overline{2}], \theta_{\mathrm{s}}=50^{\circ}, \phi_{\mathrm{s}}=210^{\circ}$ (polar angle bin is $10^{\circ}$, azimuthal angle bin is $18^{\circ}$ ). Single bounce (black solid line) and double bounce (red solid line) scattering events are shown. The probability per $2.5 \mathrm{meV}$ bin is given in the ordinate axes. The corresponding classical impact sites within the primitive cell (see Fig. 1d) for the (first) bounce are shown as insets.

and $\theta_{\mathrm{i}}=60^{\circ}, n_{\mathrm{i}}=[11 \overline{2}], \theta_{\mathrm{s}}=50^{\circ}, \phi_{\mathrm{s}}=210^{\circ}$ (Fig. 7b). The former scattering conditions may be viewed as sensitive to near parallel to surface back scattering while the latter conditions favor single bounce scattering exhibiting $100<\Delta E_{\text {loss }}<200 \mathrm{meV}$. In these figures, we show all contributions to the scattering flux. Figure 7a shows that the near parallel to surface single bounce back scattering (solid black line) is overlapped by a similar amount of double bounce scattering (dashed red line). The situation is similar in Fig. 7b. Despite the incomplete resolution of the single bounce $v s$. double bounce scattering mechanisms, it is important to note that under both of these scattering conditions, we see essentially no energy loss above $0.2 \mathrm{eV}$. For the conditions of Fig. 7a, even energy loss above $0.1 \mathrm{eV}$ is hardly seen.

This approach can be generally applied to identify other scattering mechanisms. By analysis of the trajectories, we could identify single-bounce as well as double bounce collisions where the (first) impact is near the a-top site. We could also identify single bounce collisions that impact near the hollow sites. Figure 8 shows the calculated scattering angular distributions for these three classes of collisions, integrated over all final scattering energies. One can immediately see that the three types of collisions exhibit characteristic angular distributions. 


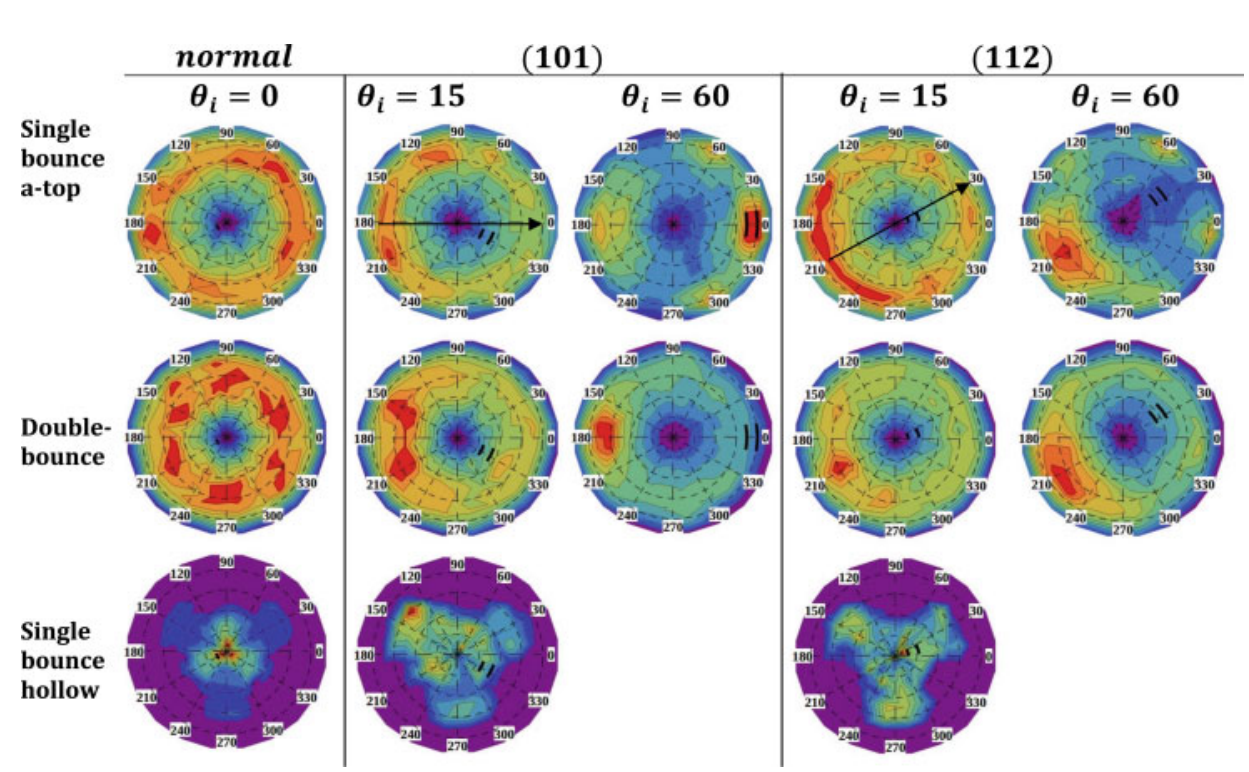

Fig. 8. Energy integrated angular distributions for non-penetrating collisions $T_{\mathrm{S}}=10 \mathrm{~K}$ and $E_{\mathrm{i}}=5 \mathrm{eV} . \theta_{\mathrm{i}}$ is the polar angle with respect to the surface normal. The polar scattering angle, $\theta_{\mathrm{s}}$, is denoted by the distance from the center of the plots. The distance between each dashed ring represents $\Delta \theta_{\mathrm{s}}=20^{\circ}$. The azimuthal scattering angle $\phi_{\mathrm{s}}$ is indicated explicitly. The azimuthal incidence angle is indicated by the arrows and by the crystallographic notation. Purple is minimum and red is maximum. Three scattering types are identified which result in characteristic scattering angular distributions.

The energy integrated angular distribution contours presented in Fig. 8 form the basis for establishing scattering conditions for which different scattering mechanisms might be resolved experimentally. To this end we have constructed the angle resolved energy loss distributions (Fig. 9a for single- (black solid and dot-dashed blue curves) and multi-bounce (red dashed curve) collisions for three specific scattering conditions. By careful choice of both incidence and scattering angles, we find conditions (albeit within the adiabatic approximation) where we predict selective detection of: 1) single bounce hollow (Fig. 9a), 2) single bounce a-top (Fig. 9b) and 3) double bounce events (Fig. 9c). In each case, the first impact site is shown in the insets of Fig. 9.

In Fig. 5 we show the angle integrated energy loss distributions for double bounce surface scattering (green dotted curves) and for all multi-bounce surface scattering (red dashed curves). For incidence angles close to normal, one sees essentially only double bounce events (red dashed and green dotted curves almost overlay one another), whereas, for glancing incidence angles, triples and higher contribute more strongly. In general, these types of collision transfer more energy to the Au lattice than do the single bounce collisions.

In order to demonstrate the usefulness of our approach, we have analyzed individual double bounce trajectories for the incidence conditions of Fig. $5 \mathrm{~d}$ : $\theta_{\mathrm{i}}=60^{\circ}, n_{\mathrm{i}}=[10 \overline{1}]$. As part of this analysis we have found the initial impact sites (Fig. 10a, the site positions are mapped into the $\mathrm{Au}(111)$ primitive cell) and the first and second impact sites connected by arrows (Fig. 10b) and classified them according to the inter-bounce 


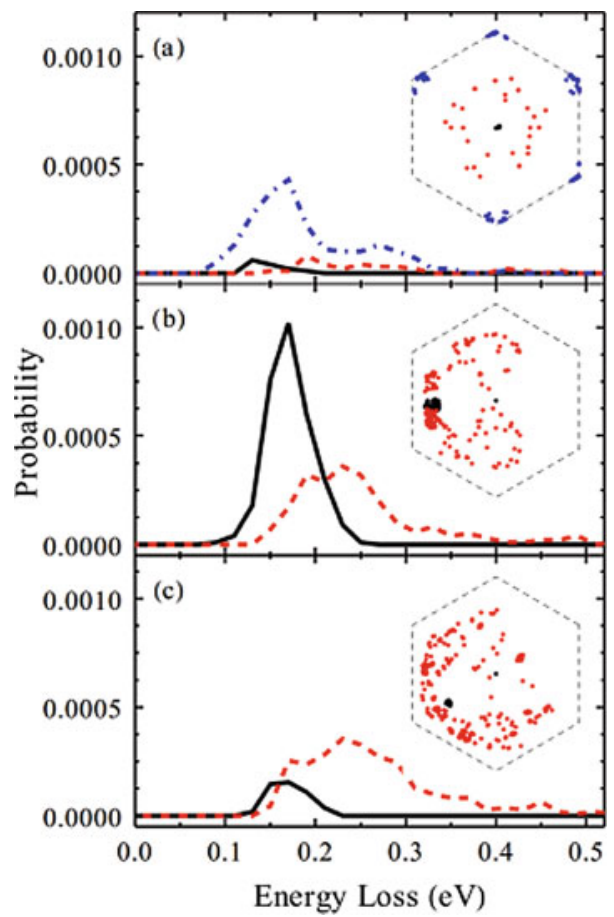

Fig. 9. H-atom impact sites (insets) and resulting translational energy loss for selected scattering angles. By choosing incidence and scattering angles one can selectively detect specific classes of scattering events. (a) $\theta_{\mathrm{i}}=0^{\circ}, \theta_{\mathrm{s}}=10^{\circ}, \phi_{\mathrm{s}}=210^{\circ}$ shows selective detection of single bounce hollow events; (b) $\theta_{\mathrm{i}}=60^{\circ}, n_{\mathrm{i}}=[10 \overline{1}], \theta_{\mathrm{s}}=75^{\circ}, \phi_{\mathrm{s}}=0^{\circ}$ shows selective detection of single bounce a-top events; (c) $\theta_{\mathrm{i}}=60^{\circ}$, $n_{\mathrm{i}}=[11 \overline{2}], \theta_{\mathrm{s}}=35^{\circ}, \phi_{\mathrm{s}}=30^{\circ}$ shows selective detection of multi-bounce collisions. Plots demonstrate selective detection of the three kinds of non-penetrating scattering. Blue dot-dashed - single bounce hollow, black solid - single bounce a-top, and red dashed - multiple bounce. Energy loss binning interval is $2.5 \mathrm{meV}$.

distance (Fig. 10c). A histogram of the inter-bounce distances shows that $65 \%$ of the double bounce events occur with nearest neighbor surface atoms (gray and blue in the color coding of Fig. 10). 10\% occur with next nearest neighbor surface gold atoms (red) due predominantly to a channeling along the [101] direction. A remarkably large number of double bounce trajectories $(25 \%)$ are long range in nature (green), where the second bounce occurs beyond the next nearest neighbor. Some of these long range doubles exhibit distances greater than $12 \AA$ between bounces. Energy integrated angular distributions of the first four peaks in the histogram are also shown as insets. One might have thought that the angular distributions for double bounce collisions would be nearly isotropic, but one sees clearly that this is not the case. Particularly surprising is the prediction (again within the adiabatic approximation) that long range double bounce scattering exhibits a narrow back scattered angular distribution. While beyond the scope of the present work, these results suggest that experimental conditions might be found where even this complex double bounce scattering could be mechanistically resolved. 


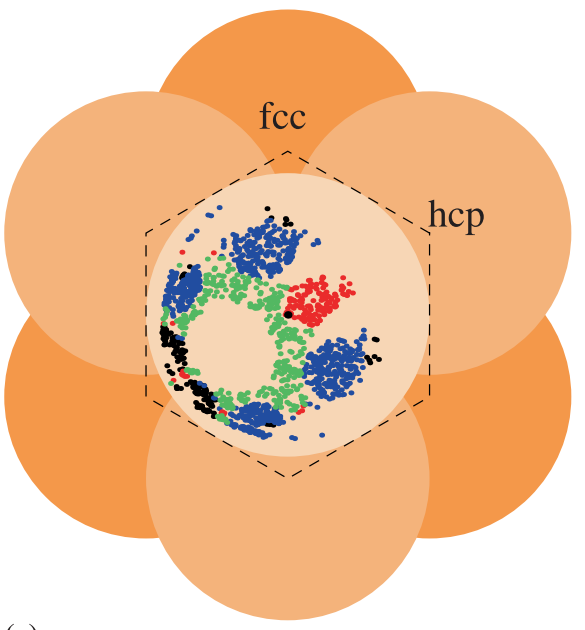

(a)

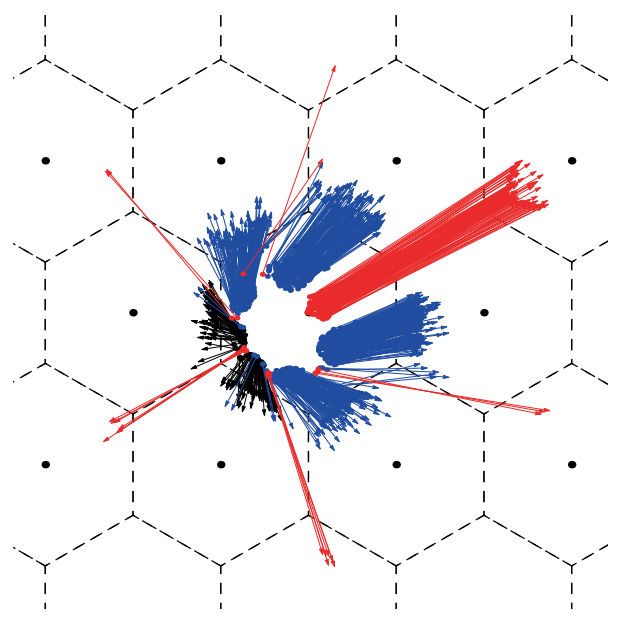

(b)

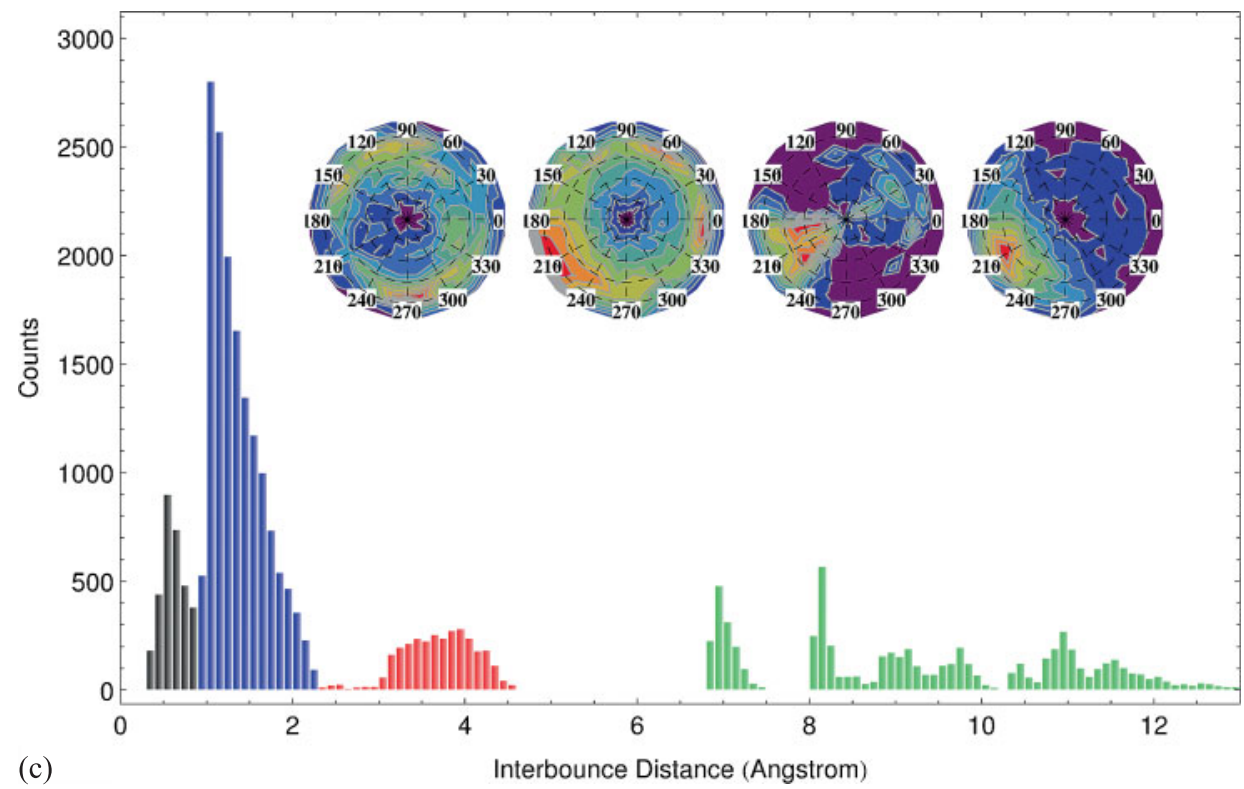

Fig. 10. Analysis of the double-bounce trajectories for $\theta_{\mathrm{i}}=60^{\circ}, n_{\mathrm{i}}=[10 \overline{1}]$. (a) Wigner-Seitz cell with the first impact sites; (b) Vectors connecting the sites of the $1^{\text {st }}$ and the $2^{\text {nd }}$ bounce. The grouping of trajectories due to the surface symmetry is clear observable; (c) A histogram of distances between impact sites. Inset to (c): Energy integrated angular distributions for the (from left to right) gray, blue, red and green doublebounce trajectories.

\section{Discussion and conclusions}

In this paper we have shown how to develop a full dimensional analytic functional form for a PES that describes the $\mathrm{H} / \mathrm{Au}$ interactions within the electronically adiabatic ap- 
proximation. This approach follows in the intellectual tradition of Eyring and Polanyi in several respects. First, we share the Eyring-Polanyi dream to reach a chemically accurate description of all the forces relevant to elementary chemical events - in this case the motion of an $\mathrm{H}$-atom in the presence of a solid metal lattice - and to carry out dynamical calculations, which allow us to visualize the atomic scale motion. This vision (originated by Eyring and Polanyi) represents a theoretical equivalent of an atomic scale microscope able to record movies on the fs time scale. To achieve this, we employ a mixture of first principles methods (DFT) and semi-empirical models to reach a useful outcome, also an approach taught by the Eyring-Polanyi work. We restrict application of this approximate PES to conditions where its weaknesses are less apparent, namely high $E_{\mathrm{i}}$ and low $T_{\mathrm{S}}$. We thus obtain results that can inform our understanding.

Even though the quality of the results of this paper is satisfactory to the purposes we set forth, they can be improved systematically in a number of ways. Future work to improve the fit to the DFT data is foreseen and underway. Future improvement will involve inclusion of AIMD trajectory based DFT data in the fit and may implement alternative fitting methods, for example, neural networks [55-57] and modified Shepard interpolation [58]. Ultimately, comparisons to experimental scattering data may help refine the PES. Despite the preliminary nature of our efforts to develop a chemically accurate full dimensional PES for the H/M interaction, the results presented for high energy low surface temperature scattering are believed to give valuable insights into the scattering of $\mathrm{H}$ from $\mathrm{Au}(111)$.

AIMD calculations, which of course require no fitting and are valid for distorted lattice structures, can serve as a quality check of this assertion. Figure 11 shows a comparison of non-penetrating contribution into the angle-integrated energy loss spectra of $\mathrm{H}$-atom scattering from $\mathrm{Au}$. The AIMD and classical MD simulations were performed as described in Sect. 2.3 with $E_{\mathrm{i}}=5 \mathrm{eV}$ and setting surface temperature to $120 \mathrm{~K}$. The incidence angles are $\theta_{\mathrm{i}}=60^{\circ}$ and $n_{\mathrm{i}}=[10 \overline{1}]$, where there is only little penetrating collisions (see Fig. 5 in case of lower $T_{\mathrm{S}}=10 \mathrm{~K}$ ). The agreement between the two methods is excellent; nevertheless the fact that the two methods arrive at slightly different energy losses is of interest and not surprising. Simulation times were limited to $120 \mathrm{fs}$ for AIMD, since AIMD calculations are computationally intensive and artifacts related to the size of the simulation cell can appear on time scales longer than this. We therefore show in Fig. 11 the part of the non-penetrating trajectories terminated at $120 \mathrm{fs}$ (blue dashed curve) for comparison. It is noteworthy that at least for the non-penetrating trajectories represented in Fig. 11, the energy loss spectrum is almost fully defined on this time scale. Of course under these incidence conditions, the role of penetrating collisions, which might require more time to re-emerge, is not large (see Table 1 and Fig. 5).

The differences between the force fields implemented by the two approaches are most likely responsible for the observed differences in the energy loss spectra. This reflects improvements that are still needed to the fitting procedure and to the treatment of lattice distortion. Nevertheless, the overall excellent agreement between two quite different methods gives us confidence that we have a semi-quantitative view of the scattering of $\mathrm{H}$ atom from $\mathrm{Au}(111)$ within the adiabatic approximation at least for the incidence conditions considered in this paper.

In summary, we have constructed a full dimensional PES for $\mathrm{H}$ atom interacting with $\mathrm{Au}(111)$. The PES is implemented for scattering calculations using classical MD. 


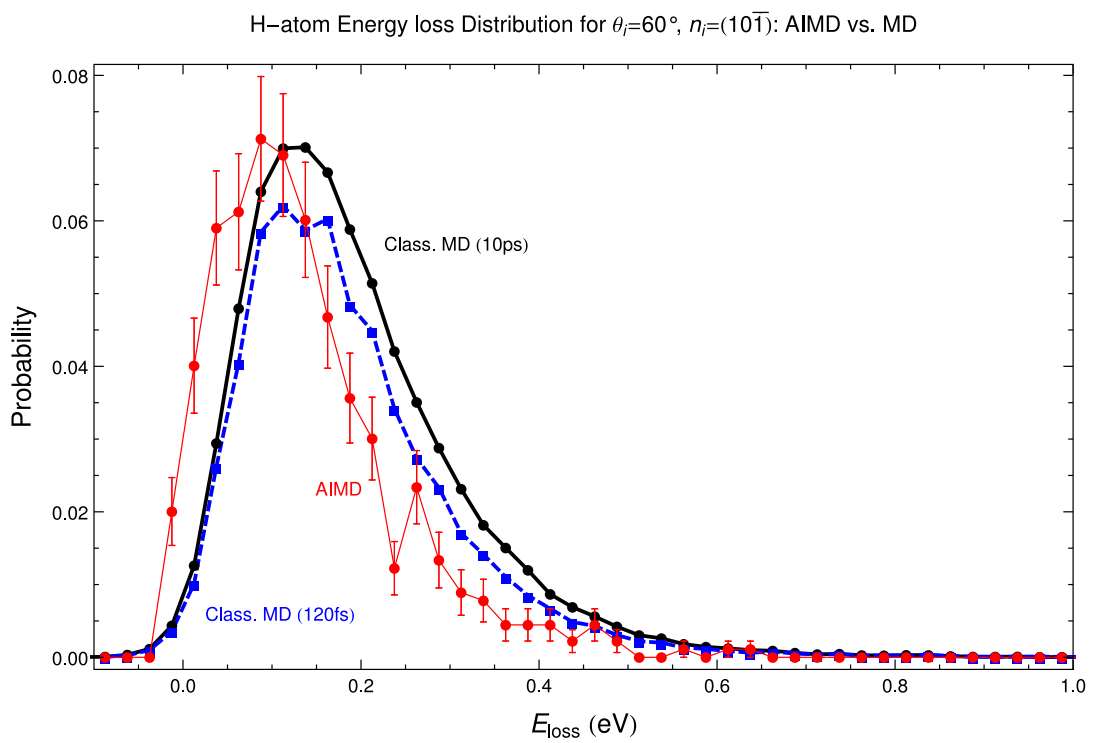

Fig. 11. Non-penetrating contribution into angle integrated energy loss probability (per binning interval of $2.5 \mathrm{meV}$ ). Ab initio Molecular Dynamics (red with statistical error bars). Classical molecular dynamics on the full-dimensional potential energy surface with simulations times of $10 \mathrm{ps}$ (black). The classical molecular dynamics results are also shown for the case when all trajectories are terminated after $120 \mathrm{fs}$ (blue). Incidence conditions are: $E_{\mathrm{i}}=5 \mathrm{eV}$ and $T_{\mathrm{S}}=120 \mathrm{~K}, \theta_{\mathrm{i}}=60^{\circ}$ and $n_{\mathrm{i}}=[10 \overline{1}]$.

The single bounce trajectories can be identified and are an important contribution to the overall scattering at all incidence conditions. They exhibit an energy loss spectrum that can be understood from the attractive Baule limit. By controlling incidence angles and detecting energy and angle resolved scattering, it appears possible to resolve three kinds of collisions: single bounce atop, single bounce hollow and multiple (predominantly double) bounce non-penetrating collisions. We see penetrating collisions, and note that these are reduced in importance at more glancing scattering angles. Back scattered single bounce trajectories travelling nearly parallel to the surface, $\theta_{\mathrm{S}}=75^{\circ}$, transfer less than $2 \%$ of the $\mathrm{H}$-atom incidence energy to $\mathrm{Au}$ atom motion. Double (and higher) bounce collisions are important at all incidence conditions examined in his work. The energy loss due to double bounce trajectories is consistent with a value twice the attractive Baule limit. We analyzed multiple bounce trajectories and find they are highly orderly. The fraction of multiple bounce collisions that occur with only two bounces is highest at normal incidence, where there are essentially no triples or higher. The majority of double bounce trajectories are $\mathrm{H}$-atom collisions with two neighboring surface gold atoms; however, a remarkably large number of double bounce trajectories $(25 \%)$ are long range in nature, where the second collision is farther away than the next nearest neighbor. Even these collisional events possess characteristic angle and energy scattering distributions that suggests they might be experimentally identified.

Finally, we emphasize that the results of these MD calculations carried out on a full dimensional semi-empirical PES should be viewed with some caution and require more 
rigorous validation than is presently possible. More detailed comparisons to AIMD results and improvements in the fitting procedure and extension of the DFT-based structural and energetic input will, we believe, eventually lead to a highly accurate full dimensional PES in the form of an analytical function. This will allow highly informative calculations with meaningful comparisons to experiments to be carried out.

\section{Acknowledgement}

The Göttingen group acknowledges support of a grant under the joint Programme ANRDFG-CHEMISTRY 2011 entitled Dynamics of Hydrogen and Deuterium on Gold and Silver Surfaces (DRAGS). We thank Mr. Stefan Ringe for writing Python scripts for some of the classical MD simulations.

\section{References}

1. J. Strömquist, L. Bengtsson, M. Persson, B. Hammer, Surf. Sci. 397 (1998) 382.

2. P. Ferrin, S. Kandoi, A. U. Nilekar, M. Mavrikakis, Surf. Sci. 606 (2012) 679.

3. M. Tomellini, React. Kinet. Catal. L. 97 (2009) 179.

4. T. Kammler, J. Kuppers, J. Chem. Phys. 111 (1999) 8115.

5. K. J. Maynard, A. D. Johnson, S. P. Daley, and S. T. Ceyer, Faraday Discuss. 91 (1991) 437.

6. G. Lee, E. W. Plummer, Surf. Sci. 498 (2002) 229.

7. X. W. Sha, B. Jackson, Chem. Phys. Lett. 357 (2002) 389.

8. K. W. Jacobsen, J. K. Nørskov, Phys. Rev Lett. 59 (1987) 2764.

9. J. K. Nørskov, B. I. Lundqvist, Surf. Sci. 89 (1979) 251.

10. H. Nienhaus, Surf. Sci. Rep. 45 (2002) 3.

11. M. Lindenblatt, J. van Heys, E. Pehlke, Surf. Sci. 600 (2006) 3624.

12. J. R. Trail, D. M. Bird, S. Holloway, J. Chem. Phys. 119 (2003) 4539.

13. J. C. Tremblay, S. Monturet, P. Saalfrank, Phys. Rev. B 81 (2010) 063420.

14. B. Gergen, H. Nienhaus, W. H. Weinberg, and E. W. McFarland, Science, 294 (2001) 2521.

15. Y. G. Li, G. Wahnström, Phys. Rev Lett. 68 (1992) 3444.

16. Y. G. Li, G. Wahnström, Phys. Rev. B 46 (1992) 14528.

17. Y. G. Li, G. Wahnström, Phys. Rev. B 51 (1995) 12233.

18. Y. G. Li, G. Wahnström, H Motion in Pd and Nb: a Molecular-Dynamics Study, in: Materials Theory and Modelling, J. Broughton, P. Bristowe, J. Newsam (Eds.), Vol. 291, Master. Res. Soc, Pittsburgh, PA, USA, 1993.

19. C. Díaz, E. Pijper, R. A. Olsen, H. F. Busnengo, D. J. Auerbach, and G.-J. Kroes, Science, 326 (2009) 832.

20. F. Nattino, C. Díaz, B. Jackson, and G.-J. Kroes, Phys. Rev Lett. 108 (2012) 236104.

21. M. S. Daw, M. I. Baskes, Phys. Rev. B 29 (1984) 6443.

22. S. M. Foiles, M. I. Baskes, M. S. Daw, Phys. Rev. B 33 (1986) 7983.

23. K. W. Jacobsen, P. Stoltze, J. K. Nørskov, Surf. Sci. 366 (1996) 394.

24. H. Eyring, M. Polanyi, Z. Phys. Chem. B 12 (1931) 279.

25. E. Lennard-Jones, T. Faraday Soc. 28 (1932) 333.

26. A. Gross, S. Wilke, M. Scheffler, Phys. Rev. Lett., 75 (1995) 2718.

27. G. J. Kroes, J. Baerends, R. C. Mowrey, Phys. Rev. Lett., 78 (1997) 3583.

28. P. Nieto, E. Pijper, D. Barredo, G. Laurent, R. A. Olsen, E. J. Baerends, G.-J. Kroes, and D. Farias, Science, 312 (2006) 86.

29. G. R. Darling, S. Holloway, Rep. Prog. Phys. 58 (1995) 1595.

30. A. Gross, Surf. Sci. Rep. 32 (1998) 291.

31. G. J. Kroes, Prog. Surf. Sci. 60 (1999) 1.

32. G. J. Kroes, M. F. Somers, J. Theor. Comput. Chem. 4 (2005) 493.

33. A. C. Luntz, Surf. Sci. 603 (2009) 1557. 
34. G. J. Kroes, Phys. Chem. Chem. Phys., 14 (2012) 14966.

35. C. Díaz, J. K. Vincent, G. P. Krishnamohan, R. A. Olsen, G.-J. Kroes, K. Honkala, and J. K. Norskov, Phys. Rev. Lett., 96 (2006) 096102.

36. M. Alducin, R. D. Muino, H. F. Busnengo, and A. Salin, Phys. Rev. Lett., 97 (2006) 056102.

37. G. A. Bocan, R. D. Muino, M. Alducin, H. F. Busnengo, and A. Salin, J. Chem. Phys., 128 (2008) 154704.

38. J. D. White, J. Chen, D. Matsiev, D. J. Auerbach, and A. M. Wodtke, Nature, 433 (2005) 503.

39. B. Baule, Ann. Phys. 44 (1914).

40. W. Kohn, L. J. Sham, Phys. Rev. A 140 (1965) 1133.

41. P. Hohenberg, W. Kohn, Phys. Rev. B 136 (1964) 864.

42. G. Kresse, J. Hafner, Phys. Rev. B 49 (1994) 14251.

43. G. Kresse, J. Hafner, Phys. Rev. B 47 (1993) 558.

44. G. Kresse, J. Furthmüller, Comp. Mater. Sci. 6 (1996) 15.

45. G. Kresse, J. Furthmüller, Phys. Rev. B 54 (1996) 11169.

46. P. Perdew, K. Burke, M. Ernzerhof, Phys. Rev. Lett., 77 (1996) 3865.

47. A. Varganov, R. M. Olson, M. S. Gordon, G. Mills, and H. Metiu, J. Chem. Phys., 120 (2004) 5169.

48. J. P. Perdew, J. A. Chevary, S. H. Vosko, K. A. Jackson, M. R. Pederson, D. J. Singh, and C. Fiolhais, Phys. Rev. B 46 (1992) 6671.

49. S. R. Bahn, K. W. Jacobsen, Comput. Sci. Eng. 4 (2002) 56.

50. L. Verlet, Phys. Rev., 165 (1968) 201.

51. L. Verlet, Phys. Rev., 159 (1967) 98.

52. M. Pavanello, D. Auerbach, A. M. Wodtke, G.-J. Kroes, in preparation, (2013).

53. J. W. Lynn, H. G. Smith, R. M. Nicklow, Phys. Rev. B 8 (1973) 3493.

54. B. Hammer, L. B. Hansen, J. K. Nørskov, Phys. Rev. B 59 (1999) 7413.

55. J. Behler, Phys. Chem. Chem. Phys., 13 (2011) 17930.

56. J. Behler, J. Chem. Phys., 134 (2011) 074106.

57. K. V. J. Jose, N. Artrith, J. Behler, J. Chem. Phys., 136 (2012) 194111.

58. T. J. Frankcombe, M. A. Collins, D. H. Zhang, J. Chem. Phys., 137 (2012) 144701. 